\title{
Population pharmacokinetics of azithromycin and chloroquine in healthy adults and paediatric malaria subjects following oral administration of fixed-dose azithromycin and chloroquine combination tablets
}

\author{
Qinying Zhao ${ }^{1 *}$, Thomas G Tensfeldt ${ }^{1}$, Richa Chandra ${ }^{2}$ and Diane R Mould ${ }^{3}$
}

\begin{abstract}
Background: Population pharmacokinetics (PK) of azithromycin (AZ) and chloroquine (CQ) following administration of fixed-dose combination tablet formulations of AZ and CQ (AZCQ) was evaluated using data from two studies: 1) in children with symptomatic uncomplicated falciparum malaria in sub-Saharan Africa; and 2) in healthy adults in the United States.

Methods: Study 1 included paediatric subjects randomized to either AZCQ or artemether-lumefantrine treatment in Cohort 1 (age 5-12 years) and Cohort 2 (age 6-59 months). Dosing of AZCQ was approximately $30 \mathrm{mg} / \mathrm{kg} \mathrm{AZ}$ and 10 mg/kg CQ once daily for 3 days (for $\geq 20 \mathrm{~kg}$ weight: AZ/CQ 300/100 mg per tablet; 5 to <20 kg weight: AZ/CQ 150/50 mg per tablet). Study 2 included adults randomized to receive either two AZCQ tablets (AZ/CQ 250/155 mg per tablet) or individual commercial tablets of AZ $500 \mathrm{mg}$ and CQ $300 \mathrm{mg}$. Serum AZ and plasma CQ concentrations from both studies were pooled. Population PK models were constructed using standard approaches to evaluate the concentration-time data for AZ and CQ and to identify any covariates predictive of PK behaviour.

Results: A three-compartment PK model with linear clearance and absorption adequately described AZ data, while a two-compartment model with linear clearance and absorption and an absorption lag adequately described CQ data. No overall bias or substantial model misspecification was evident using diagnostic plots and visual predictive checks. Body weight as an allometric function was the only covariate in the final AZ and CQ PK models. There were significantly lower AZ (0.488 vs $0.745[\mathrm{mg} \cdot \mathrm{h} / \mathrm{L}] /[\mathrm{mg} / \mathrm{kg}], \mathrm{p}<0.00001)$ and CQ $(0.836 \mathrm{vs} 1.27[\mathrm{mg} \cdot \mathrm{h} / \mathrm{L}] /[\mathrm{mg} / \mathrm{kg}]$, $\mathrm{p}<0.00001)$ exposures $\left(A \cup C_{\text {inf }}\right)$ normalized by dose $(\mathrm{mg} / \mathrm{kg})$ in children compared with the adults.

Conclusions: The PK of AZ and CQ following administration of AZCQ was well described using a three- and twocompartment model, respectively. AZ and CQ exhibited linear absorption and clearance; the model for CQ included an absorption lag. Weight was predictive of exposure for both AZ and CQ. Assuming equivalent dosing (mg/kg), AZ and CQ exposure in children would be expected to be lower than that in adults, suggesting that children may require a higher dose $(\mathrm{mg} / \mathrm{kg})$ than adults to achieve the same AZ and CQ exposure.
\end{abstract}

Keywords: Population pharmacokinetics, Malaria, Azithromycin, Chloroquine

\footnotetext{
* Correspondence: qinying.zhao@pfizer.com

'Pfizer Inc, 445 Eastern Point Road, MS8260-2112, Groton CT 06340, USA

Full list of author information is available at the end of the article
} 


\section{Background}

Worldwide, malaria remains a burden to global health. The World Health Organization reports that approximately 3.3 billion people were at risk of malaria in 2010, with the population of sub-Saharan Africa at the highest risk [1]. The combination of $\mathrm{AZ}$ and $\mathrm{CQ}$ exhibits synergistic activity against CQ-resistant strains of Plasmodium falciparum in vitro and in vivo [2-4]. Results from Phase 2 and 3 studies demonstrated that the combination of AZ and CQ had $\sim 95 \%$ or more efficacy against symptomatic uncomplicated $P$. falciparum malaria in various geographic areas $[3,5,6]$. To be useful in combination, anti-malarial agents must not have clinically significant pharmacokinetic (PK) interactions. A lack of a PK interaction between $\mathrm{AZ}$ and $\mathrm{CQ}$ has been demonstrated [7]. In healthy adults, CQ did not have any clinically relevant effect on the PK of AZ; AZ also did not have any clinically relevant effect on either the PK of CQ or its active metabolite, desethylchloroquine (DECQ).

For adults, an investigational AZCQ tablet formulation has been developed containing AZ $250 \mathrm{mg}$ and CQ $155 \mathrm{mg}$ and has been evaluated in a bioavailability study in healthy adult volunteers with intensive PK sampling [8]. In this study, the AZCQ tablet was shown to be bioequivalent to the AZ $500 \mathrm{mg}$ (Zithromax ${ }^{\circ}$, Pfizer Inc, New York, NY, USA) and CQ 300 mg (Aralen ${ }^{\circ}$, Sanofi Aventis, Bridgewater, NJ, USA) tablets taken individually in healthy adults using area under the concentrationtime curve (AUC) as the criterion of exposure. Two additional paediatric AZCQ tablet formulations (AZ $300 \mathrm{mg} / \mathrm{CQ} 150 \mathrm{mg}$ per tablet and AZ $150 \mathrm{mg} / \mathrm{CQ}$ $50 \mathrm{mg}$ per tablet) were developed and evaluated in paediatric patients with malaria in Africa using sparse PK sampling.

The population PK analysis approach can be helpful in characterizing drug concentration-time profiles and between-subject variability in the PK. In addition, these analyses are useful to identify factors that are predictive of PK variability. Therefore, this population PK analysis was performed by combining the data from the bioavailability and paediatric treatment studies to evaluate the population PK of AZ and CQ in healthy adults from US and paediatric malaria subjects from sub-Saharan Africa.

\section{Methods}

\section{Study design}

PK data from two studies were pooled and analysed. The first study was an open-label efficacy study that compared the efficacy of AZCQ versus artemether-lumefantrine (AL) for the treatment of uncomplicated $P$. falciparum malaria in children in sub-Saharan Africa. In this study, patients were enrolled in two cohorts by age (Cohort 1
[5-12 years] and Cohort 2 [6-59 months]) and randomized to either AZCQ or AL treatment. This analysis only includes data from AZCQ-treated patients. In AZCQtreated patients, dosing of AZCQ was based on weight, with patients receiving approximately $30 \mathrm{mg} / \mathrm{kg}$ of $\mathrm{AZ}$ and $10 \mathrm{mg} / \mathrm{kg}$ of CQ. AZCQ tablets were given once daily for three days based on weight $(\geq 20 \mathrm{~kg}$ weight: AZ/CQ $300 / 100 \mathrm{mg}$ strength tablet; 5 to $<20 \mathrm{~kg}$ weight: AZ/CQ 150/50 mg strength tablet).

The second study was an open-label, randomized, single dose study to estimate the bioavailability of AZCQ tablets in healthy adult volunteers in the US. Subjects in this study were randomized to receive either two AZCQ tablets (AZ/CQ 250/155 mg strength tablet; test treatment) or co-administration of individual commercial tablets of AZ $500 \mathrm{mg}$ and CQ $300 \mathrm{mg}$ (reference treatment).

\section{Blood sampling and analysis}

In the paediatric study, sparse blood samples were collected from both cohorts to determine AZ concentration in serum and $\mathrm{CQ} /$ desethylchloroquine (DECQ) concentration in plasma prior to AZCQ dosing (window: -1 to $0 \mathrm{~h}$ ) on day 0 and day 2 . On day 2, blood samples were also collected $3 \mathrm{~h}$ (window: 2-4 h) and $8 \mathrm{~h}$ (window: 6-10 h) after AZCQ dosing. In addition, blood samples were collected at a random time point on day 7 . Blood samples in the bioavailability study were collected to assess serum AZ and plasma CQ concentrations at 0 (pre dose), 0.5, 1, 2, 3, 4, 6, 8, 12, 16, 24, 36, 48, 72 and $96 \mathrm{~h}$ post dose.

PK samples from both studies were analysed in the same bioanalytical laboratories (Centero Research in Houston, TX, USA for CQ and DECQ; Bioanalytical System Ltd in Warwickshire, UK for AZ). Plasma or serum samples were analysed for serum AZ, plasma CQ and plasma DECQ concentrations using validated, sensitive, specific high-performance liquid chromatography with tandem mass spectrometry assays [8]. The assays had a lower limit of quantification of 10.0, 1.0 and $0.50 \mathrm{ng} / \mathrm{mL}$ for AZ, CQ and DECQ, respectively. In the data analysis, observations that were below the limit of quantification were excluded from the database. The assay accuracy (expressed as \% relative error: \%RE) of the quality control samples used during the sample analysis ranged from $0 \%$ to $6.1 \%$ in the paediatric study and from $-2.1 \%$ to $4.7 \%$ in the adult study for AZ, from -3.2 to $9.8 \%$ in the paediatric study and from $-0.4 \%$ to $3 \%$ in the adult study for $C Q$, and from $0.0 \%$ to $14.5 \%$ in the paediatric study for DECQ. The assay precision data were $\leq 4.9 \%$ $\mathrm{CV}$ in the paediatric study and $\leq 4.7 \%$ in the adult study for $\mathrm{AZ}, \leq 11.2 \% \mathrm{CV}$ in the paediatric study and $\leq 8.9 \%$ in the adult study for $C Q$, and $\leq 10.8 \%$ in the paediatric study for DECQ. 


\begin{tabular}{ll} 
Table $\mathbf{1}$ Covariates assessed in the pharmacokinetic \\
analysis \\
\hline Covariate (unit) & Type \\
\hline Actual dose $(\mathrm{mg})$ & Continuous \\
Study $(1$ or 2$)$ & Categorical \\
Age (years) & Continuous \\
Gender & Categorical \\
Race & Categorical \\
Weight at baseline $(\mathrm{kg})$ & Continuous \\
Height (cm) & Continuous \\
Body surface area at baseline $\left(\mathrm{m}^{2}\right)$ & Continuous \\
Body mass index at baseline $\left(\mathrm{kg} / \mathrm{m}^{2}\right)$ & Continuous \\
Ideal body weight at baseline $(\mathrm{kg})$ & Continuous \\
Lean body weight at baseline $(\mathrm{kg})$ & Continuous \\
Aspartate aminotransferase at baseline $(\mathrm{U} / \mathrm{L})$ & Continuous \\
Alanine aminotransferase at baseline $(\mathrm{U} / \mathrm{L})$ & Continuous \\
Bilirubin at baseline (mg/dL) & Continuous \\
Albumin at baseline $(\mathrm{g} / \mathrm{dL})$ & Continuous \\
Calculated creatinine clearance at baseline $(\mathrm{mL} / \mathrm{min})$ & Continuous
\end{tabular}

\section{Population PK analysis}

Concentration-time data collected in both studies were analysed using the mixed-effects modelling tool, NONMEM ${ }^{\bullet}$ (version 7, level 2; ICON Plc, Dublin, Ireland). All clearance and distribution parameters are reported as the ratio of the parameter and bioavailability since oral dosing was not accompanied by an intravenous dose. All evaluations used a log-transform both sides approach.
Population PK models were constructed to evaluate the concentration-time data for $\mathrm{AZ}$ and $\mathrm{CQ}$ and to identify any covariates predictive of $\mathrm{PK}$ behaviour using standard approaches. The structural model consisted of a compartmental PK model to describe the observed concentrationtime profiles as well as between subject variability in the PK behaviour. For both AZ and CQ, two- and threecompartment models were evaluated. In addition, a onecompartment model was also evaluated for completeness, but did not describe the data well and was therefore not used for either drug.

The covariate models in this PK model were defined to represent the shift in value of the parameter of interest to a value for a hypothetical patient. The reference patient for this analysis was 40 years of age, weighed $70 \mathrm{~kg}$ and had a body surface area of $1.73 \mathrm{~m}^{2}$ and a body mass index of $15 \mathrm{~kg} / \mathrm{m}^{2}$. Covariates examined as potential predictors of PK activity are listed in Table 1. Only those covariates that individually influenced the structural parameters were added to create the full model. The full model then underwent reduction where covariates were removed one at a time and the impact of removal was assessed (backward deletion method). The covariate was retained in the final model if its removal resulted in an increase in the objective function of $\geq 10.83$ points $(\mathrm{p}<0.001$ ) from the full model.

Two different allometric models using weight normalized PK parameters to size were evaluated [9]. The first model used an allometric coefficient of 0.75 for clearances and 1 for volumes. The second model used estimated allometric coefficients for clearances and volumes. Wherever

Table 2 Parameter estimates for final population PK model: azithromycin in serum and chloroquine in plasma

\begin{tabular}{|c|c|c|c|c|}
\hline \multirow[t]{2}{*}{ Parameter (units) } & $\begin{array}{l}\text { PK parameter } \\
\text { mean (\%RSE) }\end{array}$ & $\begin{array}{c}\text { Inter-individual } \\
\text { variability \%CV (\%RSE) }\end{array}$ & $\begin{array}{l}\text { PK parameter } \\
\text { mean (\%RSE) }\end{array}$ & $\begin{array}{c}\text { Inter-individual } \\
\text { variability \%CV (\%RSE) }\end{array}$ \\
\hline & \multicolumn{2}{|c|}{ Azithromycin } & \multicolumn{2}{|c|}{ Chloroquine } \\
\hline $\mathrm{CL} / \mathrm{F}(\mathrm{L} / \mathrm{h})$ & $100(6.1)$ & $31.3(17.6)$ & $59.1(2.3)$ & $30.5(12.2)$ \\
\hline Normalized for weight & 0.75 FIXED & & 0.75 FIXED & \\
\hline $\mathrm{V} 1 / \mathrm{F}(\mathrm{L})$ & $186(22.4)$ & $113(31.8)$ & $2,870(4.3)$ & $46.6(15.7)$ \\
\hline Normalized for weight & 1 FIXED & $C L: V 1$ covariance 0.28 & 1 FIXED & CL:V1 covariance 0.72 \\
\hline Ka $(1 / h)$ & $0.259(10.2)$ & NE & $6.12(29.4)$ & $\mathrm{NE}$ \\
\hline Q2/F (L/h) & $180(8.2)$ & NE & $61.4(11.3)$ & $\mathrm{NE}$ \\
\hline Normalized for weight & 0.75 FIXED & & 0.75 FIXED & \\
\hline $\mathrm{V} 2 / \mathrm{F}(\mathrm{L})$ & $2,890(4.8)$ & NE & $1,890(4.9)$ & $\mathrm{NE}$ \\
\hline Normalized for weight & 1 FIXED & & 1 FIXED & \\
\hline LAG (h) & NE & NE & $0.387(6.7)$ & NE \\
\hline Q3/F (L/h) & $10.6(19.0)$ & NE & $\mathrm{NE}$ & $\mathrm{NE}$ \\
\hline V3/F (L) & $2,610(46.7)$ & NE & $\mathrm{NE}$ & $\mathrm{NE}$ \\
\hline Residual error (\%RSE) & $0.406(2.4)$ & NE & $0.249(2.6)$ & NE \\
\hline
\end{tabular}

\%CV, Percent coefficient of variation; \%RSE, Relative standard error expressed as percentage; CL/F, Clearance normalized for bioavailability; IIV, Inter-individual variability; Ka, Absorption rate constant; LAG, Lag time; NE, Not estimated; PK, Pharmacokinetics; Q2/F, Inter-compartmental clearance from central to first peripheral compartment; $Q 3 / F$, Inter-compartmental clearance from central to second peripheral compartment; V1/F, Volume of distribution of the central compartment; V2/F, Volume of distribution of first peripheral compartment; $V 3 / F$, Volume of distribution of second peripheral compartment. 

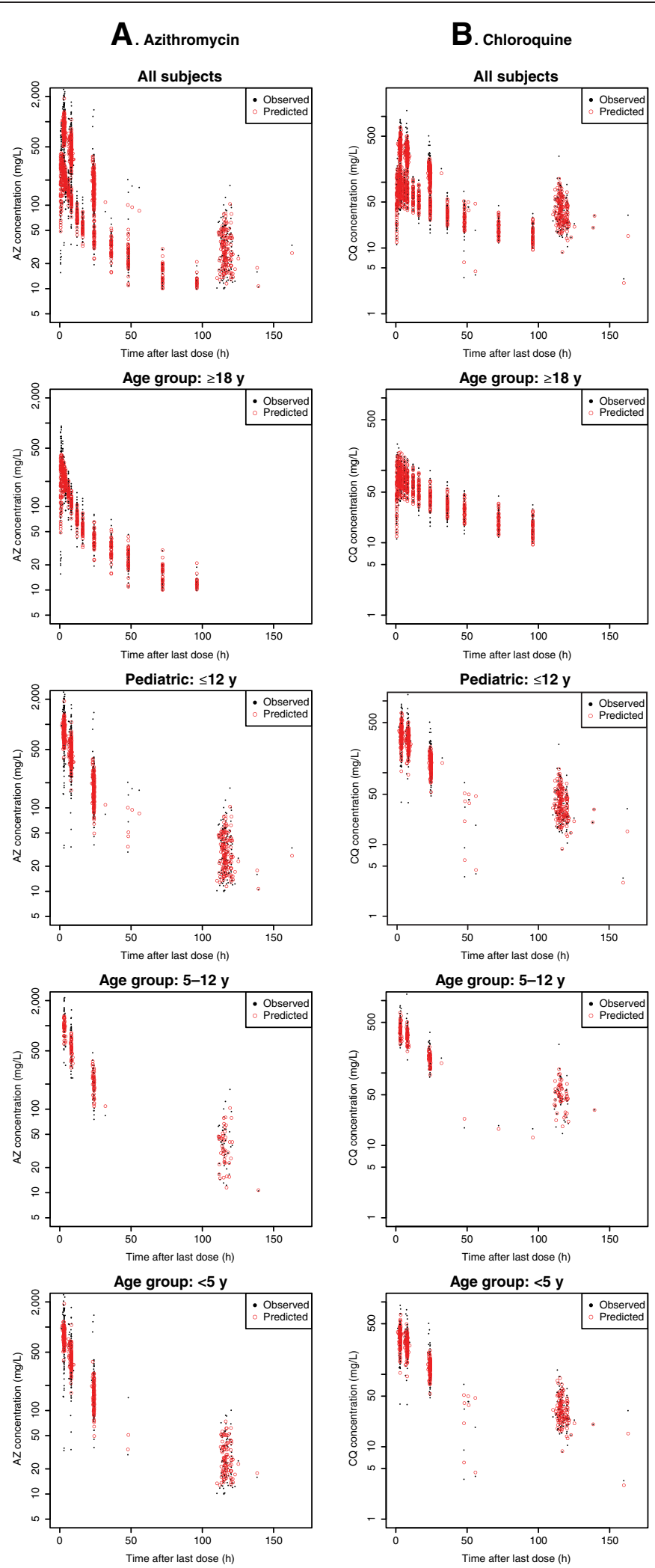

Figure 1 (See legend on next page.) 
(See figure on previous page.)

Figure 1 Final population pharmacokinetic (PK) model: predicted and observed (A) serum azithromycin (AZ) and (B) plasma chloroquine (CQ) concentrations versus time after last dose relative to sampling time. Figure 1 shows (A) serum $A Z$ and (B) plasma CQ measured and predicted concentrations in various age groups. Black filled circles represent observed data points and red open circles are population predicted values for the final PK model. Good concordance between observed and predicted data points is evident.

possible, symmetric 95\% confidence intervals were computed using the asymptotic standard errors of the parameter estimates.

\section{Results}

\section{Subject disposition and demographics}

The final dataset consisted of 1,198 and 1,197 evaluable measurable AZ and CQ concentration observations from 219 subjects including 40 adults, 123 paediatric subjects aged $<5$ years and 56 paediatric subjects aged 5 to 12 years.

\section{Models}

The final model for AZ in serum following AZCQ administration was a three-compartment model with linear clearance and absorption and no absorption lag time. The final model for CQ in plasma was a two-compartment model with linear clearance and an absorption lag time. Both models included actual body weight with fixed allometric scaling. In the AZ model, shrinkage was acceptable (defined as $\leq 20 \%$ ) for clearance (CL/F), but was high (51.8\%) for central compartment volume of distribution (V1/F) that is considered likely due to the sparse sampling in the paediatric study. In the CQ model, shrinkage was acceptable for CL/F and V1/F, but was elevated for the absorption rate constant (62\%) that could be due to the sparse sampling in the paediatric study. The condition number for the base model was less than 20 (AZ: 13.4; CQ: 5.3) for both AZ and CQ suggesting no notable colinearity. Thus, covariate evaluations on $\mathrm{CL} / \mathrm{F}$ were considered appropriate.

DECQ only had sparse concentration data from the paediatric patients and no data from the adult bioavailability study were available; thus, a population PK model for DECQ could not be identified.

\section{PK parameters}

The parameters for the final AZ and CQ population PK models are provided in Table 2. Body weight was found to be influential for both $\mathrm{AZ}$ and CQ pharmacokinetics.

Good concordance was noted between the values predicted from the PK model versus the observed concentrations of AZ and CQ (Figure 1). Goodness of fit plots also indicated that the PK models adequately described the PK data for both AZ and CQ and did not suggest substantial bias (Figure 2). A visual predicted check from all the data in the final model indicated that, for both
$\mathrm{AZ}$ and $\mathrm{CQ}$, the majority of observed concentrations fell within the predicted interquartile ranges (Figure 3).

There was no overall bias or evidence of substantial model misspecification for either AZ or CQ. Body weight as an allometric model was the only covariate in the final AZ and CQ PK models.

The dose-normalized AUC values from time 0 to infinity $\left(\mathrm{AUC}_{\mathrm{inf}}\right)$ of $\mathrm{AZ}$ and $\mathrm{CQ}$ were estimated from the respective administered doses and the individual subject clearances $(\mathrm{CL} / \mathrm{F})$ then normalized by the administered dose divided by body weight $\left(\mathrm{AUC}_{\text {inf }} /\right.$ dose $=$ dose/CL/ [dose/weight]). The paediatric population had significantly lower values than that of the adult population in the dose $(\mathrm{mg} / \mathrm{kg})$-normalized $\mathrm{AUC}_{\text {inf }}$ for both $\mathrm{AZ}(0.488$ vs $0.745 \mathrm{mg} * \mathrm{~h} / \mathrm{L} /(\mathrm{mg} / \mathrm{kg}), \mathrm{p}<0.00001)$ and CQ (0.836 vs $1.27 \mathrm{mg} / \mathrm{h} / \mathrm{L} /(\mathrm{mg} / \mathrm{kg}), \mathrm{p}<0.00001)$, indicating a lower $(\mathrm{L} / \mathrm{h} / \mathrm{kg})$ clearance value in adults as compared to paediatric subjects. Comparison of dose-normalized $\mathrm{AUC}_{\mathrm{inf}}$ for both $\mathrm{AZ}$ and CQ by age group is graphically presented in Figure 4.

\section{Discussion and conclusions}

AZ PK and CQ PK were well described using a threeand two-compartment model, respectively. These models were in general agreement with models previously published [10-13]. In the models of the current study, both $\mathrm{AZ}$ and CQ exhibited linear absorption and clearance in children and adults, but CQ showed an absorption lag. Due to the sparse PK sampling in the paediatric study, it would be difficult to identify such an absorption delay in the appearance of drug in the paediatric patients, and the ability to identify if an absorption phase exists because of the intensive sampling in the adult study. Consequently, it is not possible to determine if the lag time is the same in the paediatric patients as in the adults. Previously published values for AZ PK parameters were generally consistent with those estimated in the present analysis $[10,12]$. For $C Q$, other studies have found that a twocompartment model best fit data in women [11] and children [13]. However, neither of these models included an absorption lag or transit.

A positive correlation between age and CQ clearance was reported by Obua et al. [13], but was not confirmed in this analysis, although weight and age are highly positively correlated in paediatric subjects. However, weight was predictive of exposure for both $\mathrm{AZ}$ and $\mathrm{CQ}$ in this analysis. 

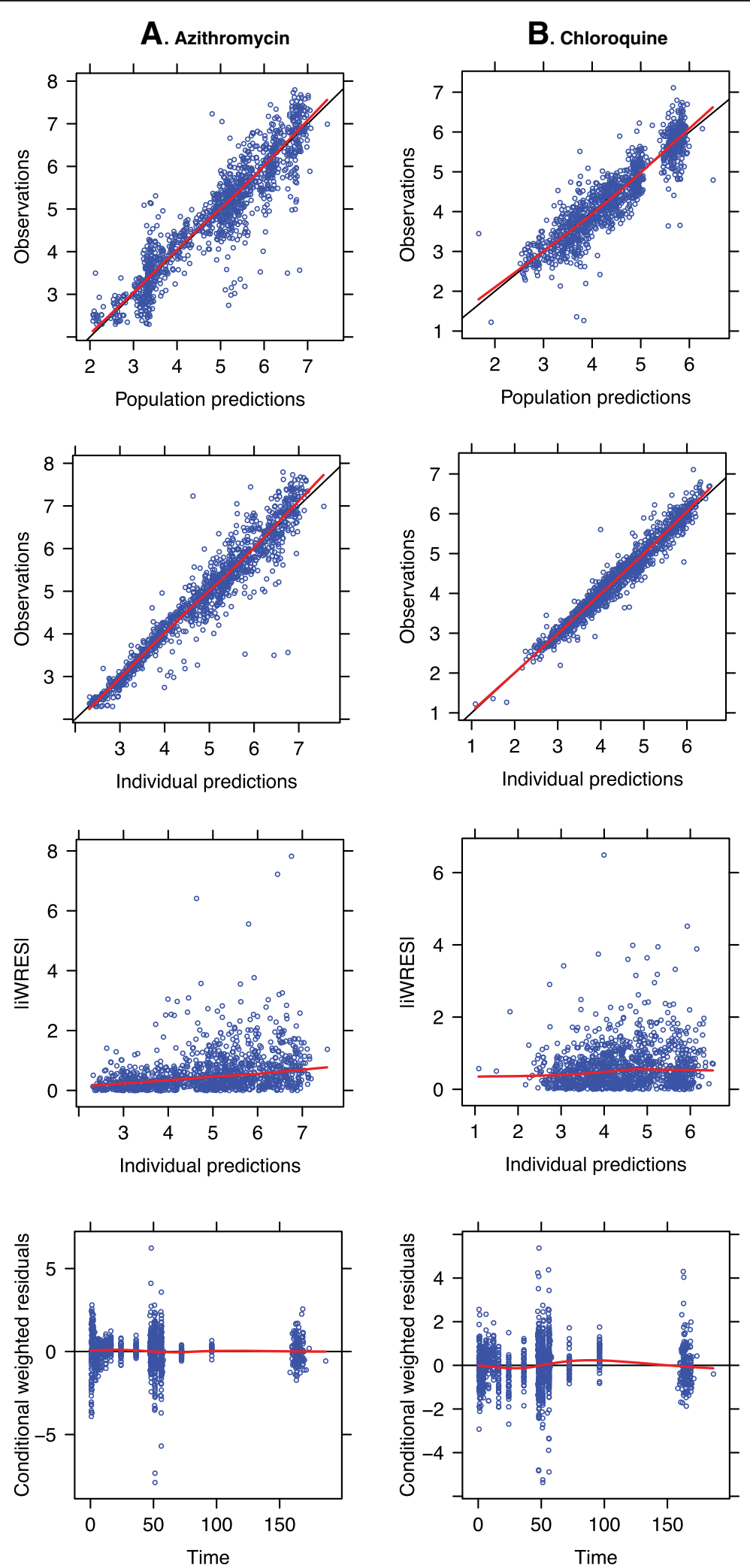

Figure 2 Goodness of fit plots for all ages for the final (A) serum azithromycin (AZ) and (B) plasma chloroquine (CQ) pharmacokinetic models. Figure 2 shows goodness of fit plots for (A) serum AZ and (B) plasma CQ measured and predicted concentrations. No systematic bias is evident and good concordance between observed and predicted concentrations is evident in the aggregate data. 

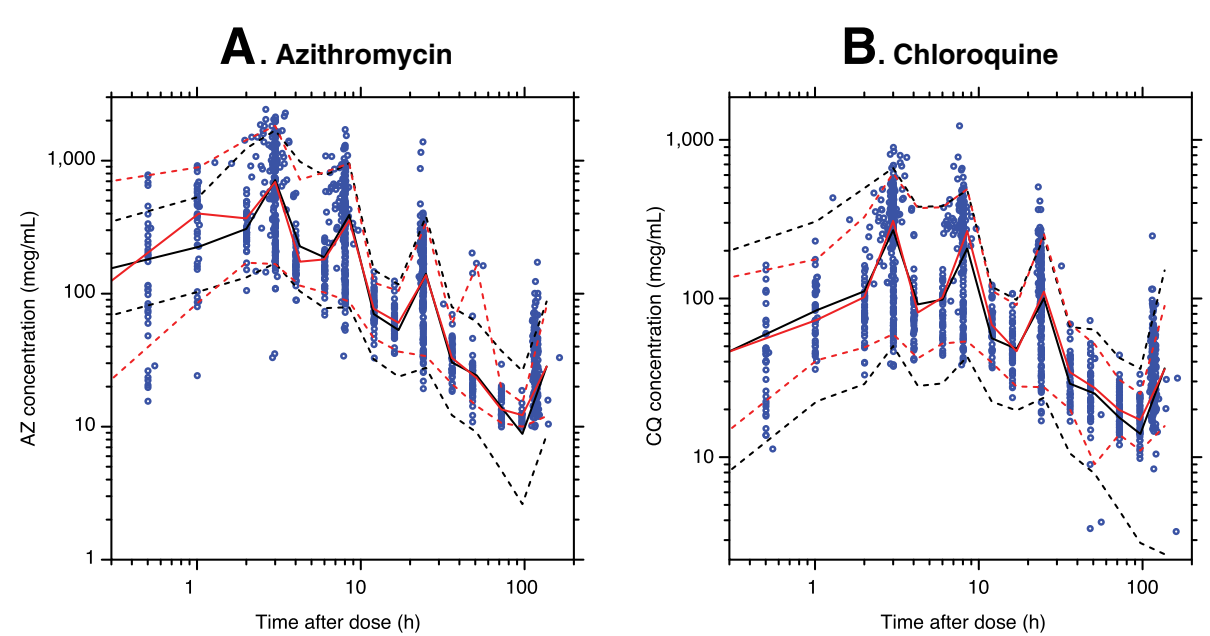

Figure 3 Visual predictive check for all concentration-time data for (A) serum azithromycin (AZ) and (B) plasma chloroquine (CQ).

Figure 3 shows the log-log plot of the visual predictive check for the final pharmacokinetic model using all data in the dataset. Open blue circles are observed concentrations. The solid black line represents the median of the predicted data (black dashed lines represent the $95 \%$ confidence interval $[\mathrm{CI}]$ ). The solid red line represents the median of the actual data (red dashed lines represent the 95\% Cl). No apparent bias was noted in the final model and overall performance was considered adequate.

Drug clearance in children was higher than that in adults when evaluated on a weight-normalized basis. Allometric models have been developed to address the higher clearance $(\mathrm{L} / \mathrm{h} / \mathrm{kg})$ observed in children $[14,15]$. Assuming equivalent dosing $(\mathrm{mg} / \mathrm{kg})$, AZ and CQ exposure in children would be expected to be lower than that in adults, suggesting that children may require a higher dose $(\mathrm{mg} / \mathrm{kg})$ than adults to achieve the same $\mathrm{AZ}$ and $\mathrm{CQ}$ exposure.

In conclusion, pharmacokinetics for $\mathrm{AZ}$ and $\mathrm{CQ}$ was well described using a three-compartment model and two-compartment model, respectively, in both healthy adult subjects and paediatric patients with symptomatic uncomplicated falciparum malaria following the full three-day course of AZCQ treatment. Both AZ and CQ exhibited linear absorption and linear clearance. In addition, there was an absorption lag in CQ. The effect of body size on PK was described using an allometric function based on normalized weight on primary AZ and CQ PK parameters. Drug clearance $(\mathrm{L} / \mathrm{h} / \mathrm{kg})$ in children was higher than that in adults when evaluated on a weight-normalized basis. This suggests that exposure (AUC) of AZ and CQ in the paediatric patients would be expected to be lower than that experienced by the adults with equal dosing on a $\mathrm{mg} / \mathrm{kg}$ basis and higher $\mathrm{mg} / \mathrm{kg}$ dosing may be required in this population.
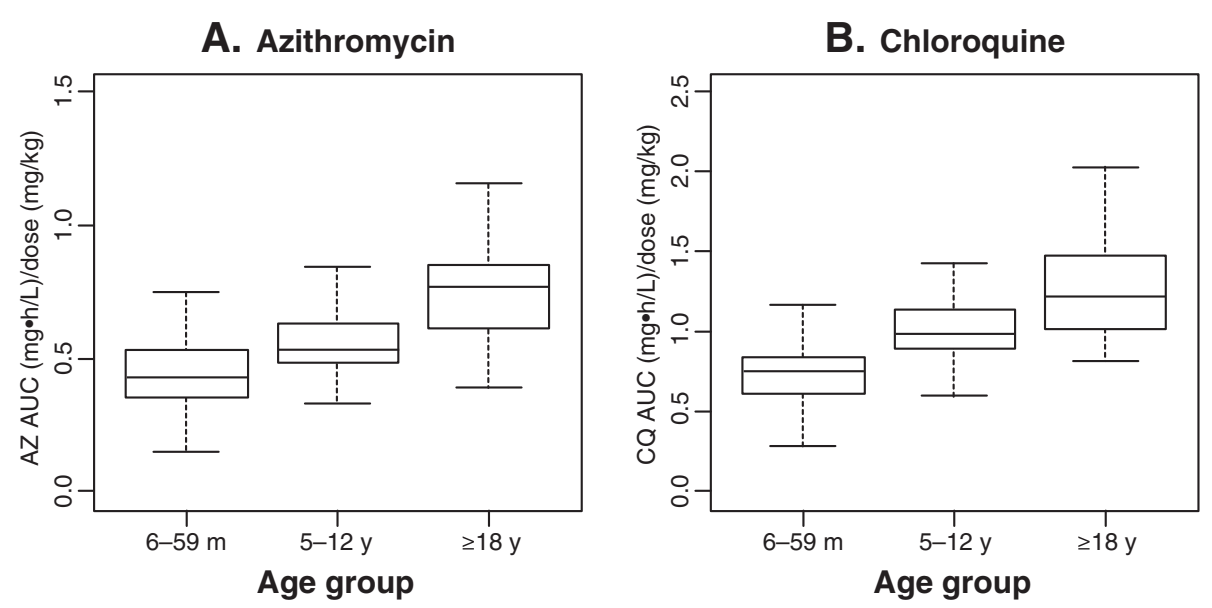

Figure 4 Comparison of dose-normalized area under the concentration-time curve $\left(A \cup C_{\text {inf }}\right)$ by age group for $(A)$ serum azithromycin $(\mathrm{AZ})$ and (B) plasma chloroquine (CQ). 


\section{Abbreviations}

$\%$ CV: Percent coefficient of variation; \%RSE: Relative standard error expressed as percentage; AL: Artemether-lumefantrine; AUC: Area under the concentration-time curve; $A \cup C_{\text {inf: }}$ Area under the concentration-time curve from time 0 to time infinity; AZ: Azithromycin; AZCQ: Fixed-dose combination tablet treatment of azithromycin and chloroquine; $C L / F$ : Clearance; $C_{\text {max: }}$ Maximum concentration; CQ: Chloroquine; DECQ: Desethylchloroquine; IVV: Inter-individual variability; Ka: Absorption rate constant; LAG: Lag time; NE: Not estimated; PK: Pharmacokinetic; Q2/F: Inter-compartmental clearance from central to first peripheral compartment; Q3/F: Inter-compartmental clearance from central to second peripheral compartment; $T_{\max }$ : Time to maximum concentration; V1/F: Volume of distribution; V2/F: Volume of distribution of first peripheral compartment; V3/F: Volume of distribution of second peripheral compartment.

\section{Competing interests}

QZ and TT are full-time employees of and own stock in Pfizer Inc. RC was a ful time employee of Pfizer Inc when these studies were conducted and owns shares in Pfizer Inc. DRM is a full-time employee of Projections Research, Inc.

\section{Authors' contributions}

All authors contributed to the development and conduct of the analysis. All authors reviewed and revised manuscript outlines and drafts and approved the final version.

\section{Acknowledgements}

This study was sponsored by Pfizer Inc. Medical writing support was provided by Linda Wagner, PharmD, of Engage Scientific Solutions and was funded by Pfizer Inc

\section{Author details}

'Pfizer Inc, 445 Eastern Point Road, MS8260-2112, Groton CT 06340, USA.

${ }^{2}$ Novartis, Cambridge, MA, USA. ${ }^{3}$ Projections Research Inc, Phoenixville, PA, USA.

Received: 5 September 2013 Accepted: 22 January 2014

Published: 29 January 2014

\section{References}

1. WHO: World malaria report 2012. Geneva: World Health Organization. http:// www.who.int/malaria/publications/world_malaria_report_2012/en/index.html.

2. Biswas S: In-vitro antimalarial activity of azithromycin against chloroquine sensitive and chloroquine resistant Plasmodium falciparum. J Postgrad Med 2001, 47:240-243.

3. Dunne MW, Singh N, Shukla M, Valecha N, Bhattacharyya PC, Dev V, Patel K, Mohapatra MK, Lakhani J, Benner R, Lele C, Patki K: A multicenter study of azithromycin, alone and in combination with chloroquine, for the treatment of acute uncomplicated Plasmodium falciparum malaria in India. J Infect Dis 2005, 191:1582-1588.

4. Ohrt C, Willingmyre GD, Lee P, Knirsch C, Milhous W: Assessment of azithromycin in combination with other antimalarial drugs against Plasmodium falciparum in vitro. Antimicrob Agents Chemother 2002, 46:2518-2524

5. Chandra R, Lewis D, Moran D, Dubhashi N, Sarkar S, Wang CS, Cai J, Dunne M: A phase 2, open label, non-comparative trial of azithromycin $2 \mathrm{~g}$ plus chloroquine $600 \mathrm{mg}$ base daily for three days for the treatment of uncomplicated Plasmodium falciparum malaria [abstract]. Am J Trop Med Hyg 2008, 79(6):374.

6. Chandra R, Lewis D, Oduro A, Mulenga M, Sagara I, Sie A, Tiono A, Dieng Y, Ogutu B, Sarkar S, Djimde A, Ansah P, Nambozi M, Zoungrana A Ouedraogo A, Fall M, Tina L, Dunne M: A phase 3, randomized open-label, comparative trial of azithromycin plus chloroquine versus mefloquine for the treatment of uncomplicated Plasmodium falciparum malaria in Africa. Presented at the 56th Annual Meeting of the American Society of Tropical Medicine and Hygiene. Philadelphia, PA; 2007.

7. Cook JA, Randinitis EJ, Bramson CR, Wesche DL: Lack of a pharmacokinetic interaction between azithromycin and chloroquine. Am J Trop Med Hyg 2006, 74:407-412.

8. Zhao Q, Puohit V, Cai J, LaBadie RR, Chandra R: Relative bioavailability of a fixed-combination tablet formulation of azithromycin and chloroquine in healthy adult subjects. J Bioequiv Availab 2013, 5:1-5. doi:10.4172/ jbb.1000127.

9. Anderson BJ, Woollard GA, Holford NH: A model for size and age changes in the pharmacokinetics of paracetamol in neonates, infants and children. Br J Clin Pharmacol 2000, 50:125-134.

10. Amsden GW, Ballow $\mathrm{CH}$, Forrest A: Comparison of the plasma, urine, and blister fluid pharmacokinetics of clarithromycin and azithromycin in normal subjects. Clin Drug Investig 1997, 13:152-161.

11. Karunajeewa HA, Salman S, Mueller I, Baiwog F, Gomorrai S, Law I, Page-Sharp M, Rogerson S, Siba P, llett KF, Davis TM: Pharmacokinetics of chloroquine and monodesethylchloroquine in pregnancy. Antimicrob Agents Chemother 2010, 54:1186-1192.

12. Liu P, Allaudeen H, Chandra R, Phillips K, Jungnik A, Breen JD, Sharma A: Comparative pharmacokinetics of azithromycin in serum and white blood cells of healthy subjects receiving a single-dose extended-release regimen versus a 3-day immediate-release regimen. Antimicrob Agents Chemother 2007, 51:103-109.

13. Obua C, Hellgren U, Ntale M, Gustafsson LL, Ogwal-Okeng JW, Gordi T, Jerling M: Population pharmacokinetics of chloroquine and sulfadoxine and treatment response in children with malaria: suggestions for an improved dose regimen. Br J Clin Pharmacol 2008, 65:493-501.

14. Anderson BJ, Holford NH: Mechanism-based concepts of size and maturity in pharmacokinetics. Annu Rev Pharmacol Toxicol 2008, 48:303-332.

15. Mahmood I: Prediction of drug clearance in children from adults: a comparison of several allometric methods. Br I Clin Pharmacol 2006, 61:545-557.

doi:10.1186/1475-2875-13-36

Cite this article as: Zhao et al:: Population pharmacokinetics of azithromycin and chloroquine in healthy adults and paediatric malaria subjects following oral administration of fixed-dose azithromycin and chloroquine combination tablets. Malaria Journal 2014 13:36.

\section{Submit your next manuscript to BioMed Central and take full advantage of:}

- Convenient online submission

- Thorough peer review

- No space constraints or color figure charges

- Immediate publication on acceptance

- Inclusion in PubMed, CAS, Scopus and Google Scholar

- Research which is freely available for redistribution
( Biomed Central 